\title{
Stuxnet detected, Pc breaks down
}

\author{
Xudong $\mathrm{Wu}$ \\ Department of Cell Biology, 2011 Collaborative Innovation Center of Tianjin for Medical Epigenetics, Tianjin Key Laboratory of Medical \\ Epigenetics, Tianjin Medical University, Tianjin 300070, China
}

Received June 26, 2016; aceepted July 2, 2016; published online July 27, 2016

Citation: Wu, X. (2016). Stuxnet detected, Pc breaks down. Sci China Life Sci 59, 1202-1203. doi: 10.1007/s11427-016-5108-4

Polycomb group $(\mathrm{PcG})$ proteins were originally identified in Drosophila. They generally maintain gene silencing by forming multimeric complexes. Two main complexes, namely Polycomb repressive complex 2 (PRC2) and PRC1, have been described. PRC2 methylates histone $\mathrm{H} 3$ on lysine 27 (H3K27). PRC1, mainly composed of Polycomb (Pc), Polyhomeotic (Ph), Posterior sex combs (Psc) and $\mathrm{dRing} /$ Sce, has been shown to directly compact chromatin in vitro. The mutation or deficiency of these members causes posterior transformation of the body plan as homeotic (HOX) genes are depressed. In the past two decades, main advances have been achieved in the diversity of PRC compositions, the dynamic chromatin targeting mechanisms, the silencing mechanisms involving both histone modifications and chromatin compaction (Blackledge et al., 2015; Simon and Kingston, 2013). Meanwhile, more and more studies have been focusing on the upstream regulatory mechanisms of PcG expression. For example, the expression of CBX proteins, the orthologs of Pc in mammalian cells have been shown to be regulated at the transcriptional and post-transcriptional levels (briefly reviewed in (Gil and O'Loghlen, 2014)). Furthermore, PcG protein stability as well as their localization or enzymatic activities has also been reported to be regulated by post-translational modifications (PTMs), such as phosphorylation and ubiquitination (Niessen et al., 2009). In a recent issue of Developmental Cell, Du et al. described an additional layer of PTM-independent regulation on $\mathrm{PcG}(\mathrm{Pc})$ stability (Du et al., 2016) (Figure 1).

Taking advantage of an engineered insertion of anten-

email: wuxudong@tmu.edu.cn na-specific Gal4 driver system to induce transcription, Du et al. performed a Gene Search-based mis-expression screen for developmental regulators located on the X chromosome in Drosophila. They identified a gene Stx, whose induction led to defective antenna development. Then the authors validated the phenotype by generating transgenic flies overexpressing Stx that displayed wing-to-haltere as well as antenna-to-leg transformation. Accordingly, high levels of Stx resulted in the de-repression of homeotic genes Antp and $U b x$, etc. Therefore, overactive Stx has a general role in homeotic transformation, which is reminiscent of the loss of PcG functions. In contrast, the loss of Stx caused early lethality at the pupal stage of development, which could be rescued by deletion of one allele of $P c$. Moreover, the authors showed that Stx and Pc display a reciprocal expression pattern. These findings strongly indicated an inverse functional relationship between Stx and Pc.

In addtion to the genetic interaction, the physical interaction between Stx and Pc was confirmed by in vivo co-immunoprecipitation and in vitro GST-pull down assays. And a Pc-binding domain $(\mathrm{PcB})$ was mapped in Stx. Interestingly, increased expression of wild type Stx but not the Stx $\Delta \mathrm{PcB}$ mutant reduced the Pc expression, suggesting that the interaction is essential for Stx to downregulate the Pc protein level. The authors then wondered whether Stx directly affects Pc protein stability. Indeed, Pc potein is subject to proteosome degradation. Overexpressing Stx facilitates Pc degradation which could be reversed by the treatment of the proteasome inhibitors. On the contrary, depletion of Stx stablizes Pc proteins. Based on these evidences, Pc breaks down at the high level of Stx. Hence the authors name the gene as Stuxnet, originally referring to a PC com- 


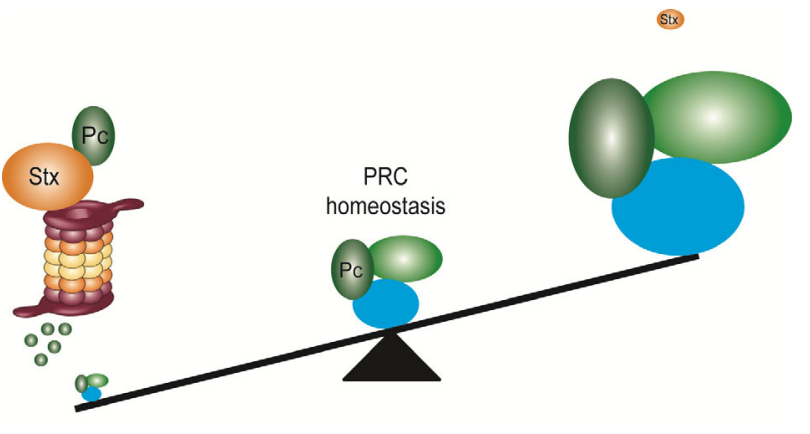

Figure 1 PRCs are dynamically regulated during development. Du et al. in a recent issue of Developmental Cell identified a new regulator Stx that controls PRC homeotastis by adapting Pc for proteasome degradation.

puter virus.

Protein ubiquitination is one of the major routes for proteasome degration. However, to our surprise, the authors found that Pc degradation is not dependent on the lysine ubiquitination despite its existence on Pc. Then what is the mechanism? After a careful analysis of the known motifs, a Ubiquitin-like (UBL) domain in Stx intrigued the researchers. Similar to other UBL-domain containing proteins, Stx interacts with the $19 \mathrm{~S}$ proteosome regulatory subunit Rpn10 in the nucleus dependent on the UBL domain. As the proteosome-interacting motif in the UBL domain forms an interacting surface with the $26 \mathrm{~S}$ proteasome, Stx relays Pc to the degradation machinery. Moreover, the ubiquitination-independent proteasome degradation of Pc was phenocopied by the in vivo genetic models in which the Stx $\Delta \mathrm{UBL}$ mutant stablized Pc protein and therefore acted in a dominant negative fashion.

Furthermore, the authors convincingly demonstrated that Stx has a negative impact of Pc functions on chromatin in vivo. As shown by both imaging and chromatin immunoprecipitation (ChIP) assays, the general decreased binding of Pc on polytene chromosomes and the engineered Polycomb Response Element (PRE) was observed upon overexpression of full length Stx, rather than the Stx $\Delta \mathrm{PcB}$ mutant. This is unlikely due to the decreased H3K27me3 levels as Stx does not seem to interact with and target PRC2 members.

Finally the authors demonstrated that this regulatory mode is conserved in mammals. For yet unknown reasons, the mouse homolog of Stx (mStx) specificially targets $\mathrm{Cbx} 4$, one of the five Pc orthologs when co-expressing in 293 cells. But it remains a mystery whether mStx targets other Cbx proteins in distinct contexts. No matter Cbx4-specific targeting or not, the pyhsiological and pathophysiological significances of this regulatory mechanism await further specification in mammals.

PcG proteins have attracted attention of more than the development biologists in the past decades, because of their documented role in regulating cellular identity. This study, combining genetic manipulations in Drosophila and biochemical approaches, has clearly illustrated a previously unrecognized role of developmental regulators in modulating PcG stability. It opened a new avenue to understand the upstream regulatory mechanisms on the precise control of PcG expression and activity. In the future experiments, it will be worthwhile to sort out the signalings that affect Stx activity and bridge Stx-Pc interaction in physiological contexts. Interestingly, the mutations or aberrant expression of Stx has been found in cancers. Stx mutations were also identified in genetic defects such as autism spectrum disorders (ASDs). Though Stx may possess Pc-independent functions, it is tempting to speculate that the $\mathrm{PcG}$ activity is disregulated due to the malfunction of Stx in these disease states.

Compliance and ethics The author(s) declare that they have no conflict of interest.

Blackledge, N.P., Rose, N.R., and Klose, R.J. (2015). Targeting Polycomb systems to regulate gene expression: modifications to a complex story. Nat Rev Mol Cell Biol 16, 643-649.

Du, J., Zhang, J., He, T., Li, Y., Su, Y., Tie, F., Liu, M., Harte, P.J., and Zhu, A.J. (2016). Stuxnet Facilitates the Degradation of Polycomb Protein during Development. Dev Cell 37, 507-519.

Gil, J., and O'Loghlen, A. (2014). PRC1 complex diversity: where is it taking us? Trends Cell Biol 24, 632-641.

Niessen, H.E., Demmers, J.A., and Voncken, J.W. (2009). Talking to chromatin: post-translational modulation of polycomb group function. Epigenetics Chromatin 2, 10.

Simon, J.A., and Kingston, R.E. (2013). Occupying chromatin: Polycomb mechanisms for getting to genomic targets, stopping transcriptional traffic, and staying put. Mol Cell 49, 808-824

Open Access This article is distributed under the terms of the Creative Commons Attribution License which permits any use, distribution, and reproduction in any medium, provided the original author(s) and source are credited. 